\title{
Phase Retrieval from Electric Field Intensity for Wide Angle Optical Fields
}

\author{
Onur Kulce, Levent Onural \\ Department of Electrical and Electronics Engineering, Bilkent University, TR-06800 Bilkent, Ankara, Turkey \\ kulce@ee.bilkent.edu.tr
}

\begin{abstract}
An intensity preserving scalar to vector electric field mapping, in a wave propagation environment, based on a filtering procedure is proposed. In a phase retrieval problem, the proposed mapping outperforms the conventional mapping.
\end{abstract}

OCIS codes: $100.5070,070.7345,260.2110$

\section{Introduction}

The aim of the phase retrieval algorithms which have been developed for both scalar and vector valued problems is to find a suitable phase pattern such that the resulting complex valued field meets some intensity criterion. In the literature, this criterion generally turns out to be the optical intensity specified over multiple parallel planes for monochromatic scalar optical fields $[1,2]$. As a result of these algorithms, the computed scalar field may end up with a wide angle field so the propagation directions of the plane wave components may lie in a large cone. If this scalar field is to be generated through some electromagnetic field source, large amount of error may arise due to the conventional scalar to vector mapping where the longitudinal component of the electric field is neglected $[3,4]$. There are also reported research results on phase retrieval under the scope of the antenna based problems where the longitudinal component of the electric field is taken into account [5-7]. In these algorithms, the intensity criterion is given in terms of the magnitude squares of the scalar components of the vector field. In this paper, the intensity is the magnitude square of the electric field vector which is given over multiple parallel planes. In the proposed algorithm, we first find a scalar field which meets the given intensity criterion using one of the phase retrieval algorithms developed for scalar fields. Then that scalar field is mapped to the vector electric field through some filtering operations such that the resulting intensity matches with the given criterion.

\section{Preliminaries and Problem Formulation}

We denote the electric field vector in three dimensional (3D) space as $\mathbf{E}(\mathbf{r})=\left[E_{x}(\mathbf{r}) E_{y}(\mathbf{r}) E_{z}(\mathbf{r})\right]^{T} \in \mathbb{C}^{3}$ where

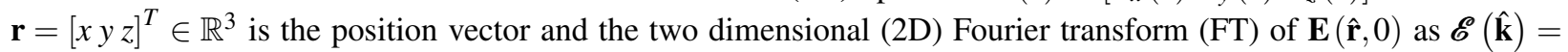

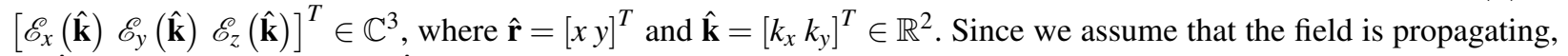
$\mathscr{E}(\hat{\mathbf{k}})$ is always zero when $|\hat{\mathbf{k}}| \geq k$, where $k$ is the wavenumber of the monochromatic field. Also, E $(\hat{\mathbf{r}}, z)$ can be found from $\mathbf{E}(\hat{\mathbf{r}}, 0)$ by using Rayleigh-Sommerfeld diffraction formulation. As a result of Gauss' Law, $\mathscr{E}_{z}(\hat{\mathbf{k}})$ should be equal to $H_{x}(\hat{\mathbf{k}}) \mathscr{E}_{x}(\hat{\mathbf{k}})+H_{y}(\hat{\mathbf{k}}) \mathscr{E}_{y}(\hat{\mathbf{k}})$, where $H_{x}(\hat{\mathbf{k}})=k_{x} /\left[k^{2}-|\hat{\mathbf{k}}|^{2}\right]^{1 / 2}$ and $H_{y}(\hat{\mathbf{k}})=k_{y} /\left[k^{2}-|\hat{\mathbf{k}}|^{2}\right]^{1 / 2}$. The electric field intensity is defined as $P(\mathbf{r})=|\mathbf{E}(\mathbf{r})|^{2}$.

If there is a relation between the $x$ and $y$ components of the electric field such that $E_{y}(\mathbf{r}) / E_{x}(\mathbf{r})=C \in \mathbb{C}$ for all $\mathbf{r}$ and if the scalar field, $S(\mathbf{r})$, is mapped to the vector field conventionally [3] as

$$
E_{x}(\mathbf{r})=\left\{\begin{array}{ll}
\frac{1}{\sqrt{1+|C|^{2}}} S(\mathbf{r}) & \text { if } C \neq \infty \\
0 & \text { if } C=\infty
\end{array} \quad, \quad E_{y}(\mathbf{r})=\left\{\begin{array}{ll}
\frac{C}{\sqrt{1+|C|^{2}}} S(\mathbf{r}) & \text { if } C \neq \infty \\
S(\mathbf{r}) & \text { if } C=\infty
\end{array},\right.\right.
$$

then, $|S(\mathbf{r})|^{2}$ becomes approximately equal to $P(\mathbf{r})$ if $S(\mathbf{r})$ is paraxial; $E_{z}(\mathbf{r})$ becomes negligibly small in this case [4]. However, if $S(\mathbf{r})$ is a wide angle field, then, the magnitude of $E_{z}(\mathbf{r})$ becomes large due to the high pass filters $H_{\{x, y\}}(\hat{\mathbf{k}})$ and the equality $|S(\mathbf{r})|^{2}=P(\mathbf{r})$ cannot be satisfied. 


\section{Scalar to Vector Field Mapping Using a Linear Shift Invariant Filter}

Here we define a filter $T_{C}(\hat{\mathbf{k}})$ for the cases $E_{y}(\mathbf{r}) / E_{x}(\mathbf{r})=C$ as

$$
T_{C}(\hat{\mathbf{k}})= \begin{cases}\frac{1}{\sqrt{|C|^{2}+1+\left|H_{x}(\hat{\mathbf{k}})+C H_{y}(\hat{\mathbf{k}})\right|^{2}}} & \text { if } C \neq \infty \text { and }|\hat{\mathbf{k}}|<k \\ \frac{1}{\sqrt{1+\left|H_{y}(\hat{\mathbf{k}})\right|^{2}}} & \text { if } C=\infty \text { and }|\hat{\mathbf{k}}|<k \\ 0 & \text { otherwise }\end{cases}
$$

Therefore, $T_{C}(\hat{\mathbf{k}})$ is a filter with a low-pass nature. Then, we assume that the vector electric field is generated from the scalar field in the Fourier domain as

$$
\mathscr{E}_{X}(\hat{\mathbf{k}})=\left\{\begin{array}{ll}
T_{C}(\hat{\mathbf{k}}) \mathscr{S}(\hat{\mathbf{k}}) & \text { if } C \neq \infty \\
0 & \text { if } C=\infty
\end{array}, \quad \mathscr{E}_{y}(\hat{\mathbf{k}})=\left\{\begin{array}{ll}
C T_{C}(\hat{\mathbf{k}}) \mathscr{S}(\hat{\mathbf{k}}) & \text { if } C \neq \infty \\
T_{C}(\hat{\mathbf{k}}) \mathscr{S}(\hat{\mathbf{k}}) & \text { if } C=\infty
\end{array},\right.\right.
$$

where $\mathscr{S}(\hat{\mathbf{k}})$ is the $2 \mathrm{D}$ FT of $S(\hat{\mathbf{r}}, 0)$. Finally, the resulting $z$ component can be computed as $H_{x}(\hat{\mathbf{k}}) \mathscr{E}_{x}(\hat{\mathbf{k}})+$ $H_{y}(\hat{\mathbf{k}}) \mathscr{E}_{y}(\hat{\mathbf{k}})$ using $\mathscr{E}_{x}(\hat{\mathbf{k}})$ and $\mathscr{E}_{y}(\hat{\mathbf{k}})$ given by Equation 3. It can be verified that if the scalar to vector mapping is carried out as given in Equation 3, the equalities

$$
|\mathscr{S}(\hat{\mathbf{k}})|^{2}=|\mathscr{E}(\hat{\mathbf{k}})|^{2} \text { and } \iint_{-\infty}^{\infty} P(\hat{\mathbf{r}}, z) \mathrm{d} \hat{\mathbf{r}}=\iint_{-\infty}^{\infty}|S(\hat{\mathbf{r}}, z)|^{2} \mathrm{~d} \hat{\mathbf{r}}
$$

are satisfied, as well, for all $\hat{\mathbf{k}}$ and $z$ values, respectively. Therefore, it can be said that the total intensity is preserved if the proposed mapping is used. Here $T_{C}(\hat{\mathbf{k}})$ can be viewed as an inverse low-pass filter which compensate the high pass effect of the filters $H_{\{x, y\}}(\hat{\mathbf{k}})$.

\section{Simulation Results}

In this section, we will compare the performances of the conventional and proposed scalar to vector mappings, that are given by Equations 1 and 3, respectively, in a phase retrieval problem for a Gaussian signal with a random phase. In order to guarantee that a solution exists to this phase retrieval algorithm, we generate the intensities at $z=0$ and $z=d$ planes from a known scalar field. We take this field, given at $z=0$ plane, as

$$
\hat{S}_{0}[n, m]=e^{-\frac{(n-N / 2)^{2}+(m-N / 2)^{2}}{2 \sigma^{2}}} e^{j \phi(n, m)},
$$

for the simulation. Here, $n \in[0, N-1]$ and $m \in[0, N-1]$ with $N=512, \sigma=64$ and $\phi(n, m)$ is a random number generated from the uniform distribution $[0, \pi / 2]$ indepently and identically. We compute the field at $z=d$ plane $\hat{S}_{d}[n, m]$, for $d=20 \mathrm{~cm}$ by using the transfer function of the Rayleigh-Sommerfeld propagation formula in 2D discrete Fourier transform (DFT) domain. We also choose the wavelength of the field as $500 \mathrm{~nm}$. The corresponding scalar optical intensities, $\left|\hat{S}_{0}[n, m]\right|^{2}=\hat{P}_{0}[n, m]$ and $\left|\hat{S}_{d}[n, m]\right|^{2}=\hat{P}_{d}[n, m]$ which are desired to be generated as the electric field intensities, can be seen in Figures 1a and 1d, respectively.

Next, by using Gerchberg-Saxton algorithm [1] and without making an approximation for the free space propagation, we compute some other scalar field such that its magnitude squares match with $\hat{P}_{0}[n, m]$ and $\hat{P}_{d}[n, m]$ at $z=0$ and $z=d$ planes, respectively. As the initial guess for $\hat{S}_{0}[n, m]$, we again assume that its phase is generated from the uniform distribution $[0, \pi / 2]$ indepently and identically. After finding appropriate scalar fields, we map them to the $x$ and $y$ components of the electric field for $C=j$, which corresponds to right hand circularly polarized field, using both the conventional and proposed methods based on the discrete versions of Equations 1 and 3, respectively. Then, the corresponding $z$ components are computed from the $x$ and $y$ components in the discrete domain, as described in [4].

Finally, for $z=0$ and $z=d$, we compute the resulting intensities $\hat{P}_{z, c o n}[n, m]$ and $\hat{P}_{z, p r o}[n, m]$ that correspond to the conventional and proposed scalar to vector mappings, respectively. In Figures $1 \mathrm{~b}$ and $1 \mathrm{e}$, the intensities as a result of the conventional mapping and in Figures $1 \mathrm{c}$ and 1f, the intensities as a result of the proposed mappings are presented for $z=0$ and $z=d$ planes. Please note that since we make the computations in DFT domain, the figures represents one period of their corresponding periodic patterns with period $n=m=512$. From the figures, it can be seen that the proposed scalar to vector mapping outperforms the conventional scalar to vector mapping in this phase retrieval problem in terms of the generation of two optical intensity patterns. For the patterns at $z=0$ plane, the excesssive amplification due to $H_{\{x, y\}}(\hat{\mathbf{k}})$ is compensated in the proposed mapping. Also, at both planes, the initial intensity patterns are preserved in the proposed mapping, whereas, in the conventional mapping, some noisy patterns appear. 


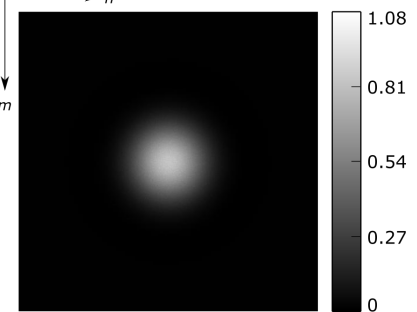

(a) $\hat{P}_{0}[n, m]$

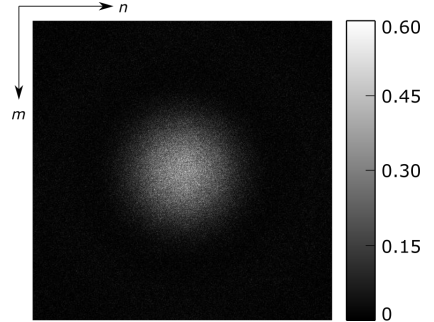

(d) $\hat{P}_{d}[n, m]$

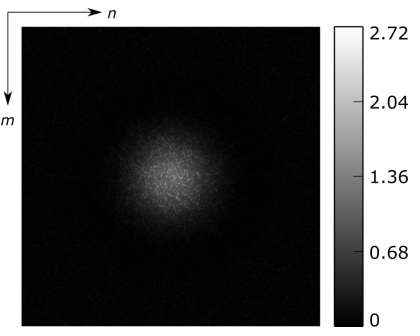

(b) $\hat{P}_{0, \text { con }}[n, m]$

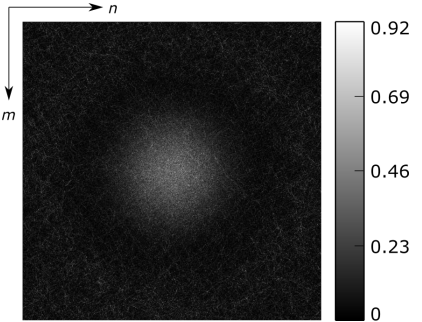

(e) $\hat{P}_{d, c o n}[n, m]$

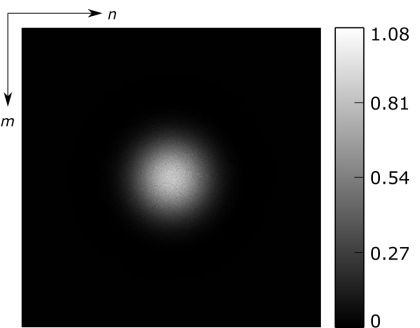

(c) $\hat{P}_{0, p r o}[n, m]$

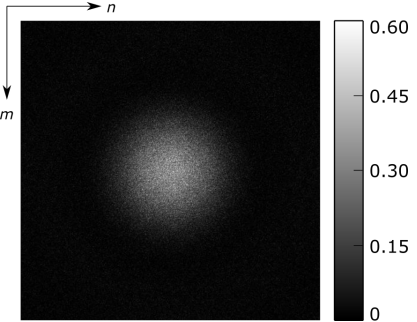

(f) $\hat{P}_{d, p r o}[n, m]$

Fig. 1: The simulation results are shown as gray scale images at $z=0$ and $z=d=20 \mathrm{~cm}$ for $N=512$. The top-left corners correspond to $(n, m)=(0,0), n$ and $m$ increase from left to right and from top to bottom, respectively. Different gray scales are used in Figures $1 \mathrm{~b}$ and $1 \mathrm{e}$, as indicated by the color bars, for the sake of visibility of the underlying Gaussian pattern which is dominated by the amplified random noise due to the uncompensated high-pass effect in the conventional procedure. The results indicate that the scalar intensity patterns are preserved if the proposed mapping is applied instead of the conventional mapping.

\section{Conclusions}

In this paper, a scalar to vector mapping using a linear shift invariant filter is proposed. As a result of this, the total scalar intensity at all $z$ planes is preserved as the electric field intensity. The proposed mapping is tested on a phase retrieval problem for a discrete Gaussian signal with a random phase and observed that the proposed mapping outperforms the conventional mapping in terms of the pointwise matching of the scalar intensity to the electric field intensity.

\section{Acknowledgements}

Onur Kulce acknowledges partial support of TÜBİTAK for this work in the form of a scholarship.

\section{References}

1. J. R. Fienup, “Phase retrieval algorithms: a comparison,” Appl. Opt. 21, 2758-2769 (1982).

2. Y. Shechtman, Y. C. Eldar, O. Cohen, H. N. Chapman, J. Miao, and M. Segev, "Phase retrieval with application to optical imaging: A contemporary overview," IEEE Signal Process. Mag. 32, 87-109 (2015).

3. O. Theimer, G. D. Wassermann, and E. Wolf, "On the foundation of the scalar diffraction theory of optical imaging," Proc. Roc. Soc. A 212, 426-437 (1952).

4. O. Kulce, L. Onural, and H. M. Ozaktas, "Evaluation of the validity of the scalar approximation in optical wave propagation using a systems approach and an accurate digital electromagnetic model," J. Mod. Opt. 63, 2382-2391 (2016).

5. M. Johansson, H. S. Lui, J. C. Bolomey, and M. Persson, "Source modeling using phaseless low-frequency near-field measurements," IEEE Trans. Electromagn. Compat. 54, 613-624 (2012).

6. T. Isernia, G. Leone, and R. Pierri, "Radiation pattern evaluation from near-field intensities on planes," IEEE Trans. Antennas Propag. 44, 701- (1996).

7. F. Las-Heras and T. K. Sarkar, "A direct optimization approach for source reconstruction and NF-FF transformation using amplitude-only data," IEEE Trans. Antennas Propag. 50, 500-510 (2002). 